\title{
Terapia de reposição hormonal e desempenho cognitivo na terceira idade 1
}

\author{
Hormone replacement therapy and cognitive \\ performance in old age
}

\author{
Ana Letícia CAMARGOS \\ Elizabeth do NASCIMENTO
}

\begin{abstract}
Resumo
No decorrer do seu ciclo de vida, as mulheres passam por uma fase denominada climatério. A terapia de reposição hormonal é o tratamento indicado para alívio dos sintomas típicos desta fase. Recentemente, descobriu-se que a terapia de reposição hormonal pode trazer benefícios adicionais, os quais se referem a proteger as mulheres contra o declínio cognitivo associado ao envelhecimento. Este artigo aborda esta questão. Embora vários estudos indiquem que o estrógeno tem efeitos positivos sobre a cognição, os resultados reportados na literatura ainda são insuficientes para indicar a terapia de reposição hormonal para a prevenção do declínio cognitivo associado ao envelhecimento. São necessárias mais pesquisas com o intuito de elucidar os possíveis benefícios desta terapia, a influência dos hormônios no envelhecimento e outros fatores associados à menopausa e à terapia de reposição hormonal que possam também influenciar a cognição.
\end{abstract}

Unitermos: Envelhecimento. Terapia de reposição de hormônios. Processos cognitivos.

\begin{abstract}
Over the course of the life-cycle of a woman, she suffers from the effects of the menopause. Hormone replacement therapy is recommended as a treatment for post-menopausal symptoms. Recently, some studies have indicated that hormone replacement therapy could modify aging-related changes in cognitive decline. The aim of this essay was to investigate the effects of hormone replacement therapy on cognitive performance. Multiple studies indicate that estrogen has positive effects on cognition. However, these data are not sufficient to recommend the use of hormone replacement therapy to prevent aging-related cognitive decline. Further research will be necessary to elucidate the benefits of hormone replacement therapy, hormone contributions and other factors related to menopause and hormone replacement therapy that might influence cognitive performance.
\end{abstract}

Uniterms: Aging. Hormone replacement therapy. Cognitive processes.

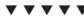

1 Artigo elaborado a partir de dissertação de A.L. CAMARGOS, intitulada "Menopausa, terapia de reposição hormonal e desempenho intelectual: um estudo transversal". Universidade Federal de Minas Gerais.

2 Universidade Presidente Antônio Carlos, Faculdade Regional de Ciências Exatas e Sociais de Barbacena, Curso de Psicologia. Rod. Deputado Zezinho Bonifácio, Km12, Colônia Rodrigo Silva, 36200-000, Barbacena, MG, Brasil. Correspondência para/Correspondence to: A.L. CAMARGOS. E-mail: <analeticiacamargos@yahoo.com.br>.

3 Universidade Federal de Minas Gerais, Faculdade de Filosofia e Ciências Humanas, Departamento de Psicologia. Belo Horizonte, MG, Brasil. 
A população mundial vem passando por um intenso processo de transição demográfica, caracterizado pelo aumento da população idosa (Liberman, 2006). O crescimento elevado da população idosa em relação aos demais grupos etários é um fenômeno mundial. Vários fatores são responsáveis por esse fenômeno, dentre eles, a baixa taxa de fecundidade, a queda na mortalidade infantil, as melhores condições de saneamento e infraestrutura básica e a redução da mortalidade em decorrência das melhores condições de saúde e dos avanços na medicina e na tecnologia (Camarano, 2006; Rodrigues \& Rauth, 2006).

Apesar de ser um fenômeno global, nos países em desenvolvimento o processo de envelhecimento populacional tem sido mais rápido e intenso. No Brasil, a expectativa de vida ao nascer aumentou cerca de nove anos entre 1980 e 2000, e os ganhos foram mais expressivos para as mulheres, que apresentaram no ano 2000 uma esperança de vida ao nascer superior em 8,4 anos à masculina (Camarano, 2006; Rodrigues \& Rauth, 2006).

Embora sejam concebidos mais homens que mulheres, em todo o mundo elas vivem cerca de quatro a dez anos a mais que eles. Esta discrepância torna-se mais acentuada entre os mais idosos, pois, contando-se os centenários de todo o mundo, são nove mulheres para cada homem (Perls \& Fretts, 2007; Spirduso, 2005).

Os índices de mortalidade entre as mulheres são mais baixos do que entre os homens, em todas as idades. No entanto, essa diferença é mais pronunciada em certos estágios da vida. Dos 15 aos 24 anos existe um surto de mortalidade masculina, a maior parte causada por comportamento negligente ou violência. Já na meia-idade, as doenças cardíacas são a principal causa das diferenças de mortalidade entre os gêneros. Nos homens, o risco de doenças cardíacas aumenta a partir dos 40 anos, já entre as mulheres este risco só começa a se elevar depois da menopausa, por volta dos 50 anos (Perls \& Fretts, 2007; Spirduso, 2005).

Não existem respostas definitivas para essas diferenças, mas foram propostas diversas explicações para entender por que as mulheres vivem mais. As explicações sociais estão relacionadas aos papéis sociais: os homens estão mais sujeitos a mortes causadas por guerras, homicídios e acidentes, têm mais hábito de fumar, consumir bebidas alcoólicas e drogas; as mulhe- res procuram mais o sistema de saúde, fazem exames e vão ao médico logo que um sintoma aparece. Podem-se citar também as explicações hormonais: o estrógeno é conhecido por proteger contra doenças cardíacas, diminuindo o mau colesterol Lipoproteína de Baixa Densidade (LDL) e elevando o bom colesterol Lipoproteína de Alta Densidade (HDL), enquanto os andrógenos atuam inversamente. A influência que os andrógenos exercem sobre o metabolismo do colesterol, diminuindo o HDL e aumentando o LDL, poderia explicar o fato de os homens serem mais suscetíveis a doenças cardíacas. Ainda, o pico de mortalidade masculina na puberdade coincide com a produção maior de testosterona (Chaimowicz, 2006; Spirduso, 2005; Perls \& Fretts, 2007).

Pode-se observar que a população idosa aumenta de maneira expressiva em relação às demais faixas etárias. Portanto, o envelhecimento populacional é uma realidade mundial, assim como o fato de as mulheres viverem mais que os homens. A feminização da velhice chama a atenção para as singularidades do ciclo da vida e do envelhecimento feminino, que são abordadas neste artigo.

\section{Desempenho intelectual e envelhecimento}

A inteligência é definida por Colom (2006) como uma capacidade mental muito geral que permite raciocinar, aprender, planejar, resolver problemas, pensar de modo abstrato e compreender ideias complexas. No entanto, a inteligência não é algo tão simples de definir. Na verdade, esse construto não desfruta de consenso mesmo entre os especialistas na área. E, embora hoje já se saiba bastante sobre a inteligência, várias questões permanecem complexas, e em muitos casos ainda não resolvidas (Almeida, 2002; Neisser et al., 1996; Rozestraten, 2002).

Sabe-se que a inteligência está por trás de muitas condutas da vida cotidiana. Nenhum traço psicológico se correlaciona com tantos fenômenos sociais: nível socioeconômico alcançado, rendimento acadêmico, desempenho no trabalho e até mesmo saúde e longevidade (Colom, 2006).

A idade é uma das variáveis mais importantes no estudo do desempenho intelectual (Nascimento, 2000). O envelhecimento acarreta um declínio normal na cognição. No entanto, vários fatores além da idade 
estão envolvidos no declínio intelectual ao longo do ciclo da vida, como nível educacional, ambiente estimulante, sucesso na vida e saúde. Para manter um bom desempenho cognitivo é importante ter interesse em aprender coisas novas, manter-se informado frente às atualidades e praticar alguma atividade física (Neri, 2006; Rozestraten, 2002).

A teoria desenvolvida por Raymond B. Cattell, em 1941, fundamenta atualmente, a maior parte das pesquisas sobre o desenvolvimento intelectual ao longo do ciclo da vida e na velhice. Cattell elaborou um modelo sobre a estrutura da inteligência composto por 19 fatores primários e cinco secundários (Flores-Mendoza \& Nascimento, 2001). Dentre os secundários, dois são considerados os mais importantes: a inteligência fluida (Gf) e a inteligência cristalizada (Gc). A inteligência fluida, de base fisiológica, reflete a capacidade de adaptação a problemas novos sem necessidade de experiências prévias, enquanto a inteligência cristalizada, de base experiencial e educacional, envolve aprendizados anteriores. Ambas as inteligências são interdependentes. $\mathrm{O}$ adjetivo fluido se refere à propriedade de fluir e o adjetivo cristalizado se refere ao corpo sólido. Então, existiria uma inteligência fluida, e a inteligência cristalizada seria resultante dos efeitos da educação e do ambiente que atuam sobre a primeira (Andrés-Pueyo, 2006; Neri, 2006).

A inteligência fluida é mais afetada pelo envelhecimento, enquanto a cristalizada é constante e pode até melhorar com o tempo. As perdas na inteligência fluida estão relacionadas às perdas sensoriais, à diminuição na velocidade de processamento da informação e às mudanças neurológicas típicas do envelhecimento. A manutenção das habilidades cristalizadas depende da influência cultural (Andrés-Pueyo, 2006; Neri, 2006; Rozestraten, 2002).

Apesar de a inteligência ser um construto muito investigado dentro da psicologia, há muito ainda a ser explorado. Durante o processo de envelhecimento ocorre um declínio normal na cognição. A idade é muito importante na compreensão desse declínio, mas existem outros fatores relacionados. Recentemente, o papel dos hormônios sexuais no desempenho intelectual ao longo do ciclo da vida vem sendo estudado, sobretudo em relação às mulheres, que vivenciam transformações mais expressivas: oscilações hormonais mensais durante a juventude e climatério/menopausa na meia-idade.

\section{Terapia de reposição hormonal e desempenho cognitivo}

Homens e mulheres apresentam diferenças em habilidades cognitivas específicas. Normalmente, os homens se sobressaem em habilidades visoespaciais, quantitativas e motoras (movimentos de grande amplitude). As mulheres se saem melhor em tarefas que utilizam habilidades verbais, velocidade perceptual e habilidade motora fina, ou seja, movimentos manuais de pequena amplitude (Haskell, Richardson \& Horwitz, 1997; Kimura, 2002; Neisser et al., 1996; Stahl, 2002).

As causas de tais diferenças podem estar relacionadas à longa história evolucionária humana, aos papéis sociais e aos hormônios sexuais. Os esteroides sexuais têm muitos efeitos no cérebro ao longo do ciclo da vida. Durante o desenvolvimento fetal, os hormônios reprodutivos influenciam a organização cerebral e, na idade adulta, seus níveis circulantes contribuem para a diferenciação entre homens e mulheres. Tais efeitos têm sido investigados durante o ciclo menstrual, devido às flutuações hormonais naturais que ocorrem mensalmente nas mulheres. Durante as fases em que o nível de estrógeno é mais alto, elas se saem melhor em tarefas de fluência verbal e habilidade motora fina e pior em tarefas espaciais. Então, variações no nível do estrógeno parecem afetar seletivamente o padrão cognitivo. Após a menopausa, as mulheres experimentam um declínio na produção dos hormônios ovarianos. Nas últimas décadas, as mulheres comumente fazem Terapia de Reposição Hormonal (TRH). Tal tratamento parece influenciar o desempenho das mulheres em algumas tarefas cognitivas, mas ainda não está claro se seus efeitos benéficos são imediatos, ou seja, apenas durante o tratamento, ou se eles se mantêm por longo prazo (Haskell et al., 1997; Kimura, 2002; McEwen, Alves, Bulloch \&Weiland, 1997; Stahl, 2002).

O emprego da TRH teve início na Alemanha em meados de 1937 e popularizou-se nos Estados Unidos a partir de 1950. O objetivo do tratamento era melhorar a qualidade de vida das mulheres na pós-menopausa. Desde então, publicações passaram a divulgar que, além de prevenir e tratar os sintomas comuns da menopausa, 
seu uso proporcionaria benefícios adicionais (Piato, 2005).

Desde que a TRH se popularizou, seus efeitos adicionais estão sendo estudados. O primeiro ensaio clínico randomizado que envolveu a administração de estrógeno ou placebo foi realizado por Caldwell e Watson em 1952 (Sherwin, 2006) com mulheres de 75 anos de idade que viviam em uma casa de repouso. Os resultados apontaram melhora na Escala Verbal, mas não na Escala de Execução da Wechsler Bellevue Intelligence Scale.

Atualmente, há um grande interesse científico quanto aos possíveis benefícios da TRH para a mulher idosa na prevenção e tratamento de déficits cognitivos, alterações do humor e doença de Alzheimer. Os resultados dos trabalhos são conflitantes, mas sugerem que o estrógeno possa manter e até melhorar as funções cognitivas em pacientes normais e dementes (D.R.B. Magalhães, Hanan \& E.B. Magalhães, 2001).

É muito comum as mulheres se queixarem de declínio cognitivo durante a menopausa, e a TRH frequentemente melhora esses sintomas. Se a diminuição dos níveis de estrógeno afeta negativamente a cognição durante a perimenopausa, então, em longo prazo, essa deficiência poderá ter um resultado mais expressivo no declínio cognitivo e também no desenvolvimento de demências (Haskell et al., 1997).

É biologicamente plausível que os hormônios possam afetar as funções cognitivas e proteger contra o declínio cognitivo associado à idade, pois existem receptores de estrógeno e progesterona em grande parte do tecido cerebral. Além do hipotálamo, que controla a função reprodutiva, eles também são encontrados na amídala, hipocampo, locus ceruleos e lobo frontal. A deficiência de estrógeno afeta o metabolismo dos neurotransmissores serotonina, dopamina, noradrenalina e acetilcolina, prejudicando a comunicação entre os neurônios. Em geral, depois da menopausa ocorre um decréscimo da eficiência cognitiva; uma parte dessa mudança é atribuída à idade, mas também pode estar relacionada às mudanças endocrinológicas (Erkkola, 1996; Maki \& Hogervorst, 2003; McEwen et al., 1997).

Haskell et al. (1997) revisaram 19 estudos: dos dez ensaios clínicos, oito apresentaram efeitos positivos 440 do estrógeno sobre a cognição e, dos nove estudos de observação (longitudinais e transversais), cinco encontraram resultados positivos, em especial na memória e na atenção. Segundo os autores, a extrema heterogeneidade das pesquisas dificulta generalizações mais contundentes; contudo, pode-se concluir que os estudos revisados fortalecem a hipótese de que o estrógeno possa melhorar a função cognitiva e reduzir o risco de demência.

Vinte e sete estudos transversais e longitudinais foram revisados por Maki e Hogervorst (2003). Destes, 21 indicaram efeitos positivos sobre o funcionamento cognitivo. Foram revisados, também, 17 ensaios clínicos, dos quais nove apresentaram efeitos benéficos, enquanto oito não apresentaram efeitos sobre a cognição. Os achados se referem a benefícios na memória e no raciocínio verbal, na velocidade da atenção, nas funções executivas, na memória figural e no status mental. Novamente, as autoras concluem que os estudos revisados são heterogêneos na medida em que usam diferentes preparações hormonais, dosagens e vias de administração, e as amostras são tão variadas em relação à idade das mulheres que dificultam qualquer conclusão mais pertinente. Desta maneira, os efeitos da TRH sobre o declínio cognitivo relacionado à idade ainda precisam ser mais bem determinados.

Verghese et al. (2000) realizaram um estudo com 35 mulheres a partir de 65 anos de idade, cirurgicamente menopausadas, sendo que dez receberam Terapia de Reposição de Estrógeno (TRE) no máximo três meses após a cirurgia e 25 nunca fizeram tal tratamento. A média de duração da TRE foi de 20,8 anos (desvio-padrão de 13,8). Os dois grupos não apresentaram diferenças, antes do início do tratamento, em relação à idade, à educação e ao QI avaliado pela Wechsler Adult Intelligence Scale-Revised (WAIS-R). No que se refere ao desempenho nos subtestes Memory Phrase (que avalia memória verbal) do Blessed Information-Memory-Concentration Test (BIMC) e Cubos do WAIS-R (que avalia habilidade construtiva), as mulheres tratadas se saíram melhor. Tal efeito permaneceu após o término do tratamento, ou seja, manteve-se por longo prazo, mesmo que o período de exposição à TRE tenha sido curto. Os autores apontam que, para a ocorrência de tal efeito, o tratamento deve ser iniciado logo após a cirurgia de retirada dos ovários, devido ao chamado período crítico. 
Existem evidências, embora não definitivas, de que o início da TRH na idade da menopausa (próximo aos 50 anos) ou logo depois da retirada dos ovários possa proteger contra o declínio cognitivo e o desenvolvimento da doença de Alzheimer. Por outro lado, a administração hormonal com atraso (depois dos 65 anos) tem pouco ou nenhum efeito benéfico sobre a cognição. Com isto, a idade do início do tratamento seria um determinante dos resultados positivos relacionados à cognição, ou seja, ela funcionaria como uma janela de oportunidade ou período crítico. A hipótese é que os neurônios se tornam menos sensíveis ao estrógeno depois de um prolongado tempo de ausência de exposição ao hormônio. Parece haver também um período específico (uma faixa de idade) para que os efeitos benéficos da TRH sobre a cognição sejam aparentes. Possivelmente, os receptores de estrógeno no sistema nervoso central sejam dependentes da idade, sendo que os neurônios mais velhos tornam-se menos responsivos ao hormônio (Maki, 2006; Maki \& Hogervorst, 2003; Sherwin, 2005, 2006).

Sherwin (2006) revisou seis ensaios clínicos. Destes, três mostraram resultados positivos sobre a cognição. Em dois deles, a média de idade das mulheres da amostra era de 45 anos e o início da terapia estrogênica ocorreu logo após a cirurgia de retirada dos ovários. Já nos estudos em que não foi encontrada relação entre aTRH e o desempenho intelectual, o estrógeno foi administrado muitos anos depois da menopausa cirúrgica ou em mulheres mais velhas (média de 65 anos). Tais achados fortalecem a hipótese de que a TRH protege contra o envelhecimento cognitivo apenas quando iniciada na idade da menopausa. Ainda, os três ensaios clínicos que apresentaram resultados benéficos sobre a cognição usaram estradiol (E2) por via intramuscular ou transdermal, e os que não encontraram efeito usaram Estrogênios Equinos Conjugados (EEC) por via oral. Isso sugere uma melhor eficácia do E2 em relação aos EEC e uma possível superioridade da via de administração não oral no que tange aos efeitos no cérebro. Os resultados desses ensaios clínicos apontam evidências de melhora na memória verbal de curto e longo prazo, memória visual e habilidades espaciais. Para a autora, o tipo de estrógeno, a dosagem e a via de administração são importantes para influenciar as funções cognitivas em mulheres na pós-menopausa e necessitam de maiores estudos.
Existem evidências de que os estrogênios também exercem influência sobre o humor, potencializando o efeito de substâncias antidepressivas. As mulheres sofrem muito mais de depressão que os homens. Contudo, durante o ciclo de vida, dois períodos são de maior risco para a depressão: o puerpério, quando os níveis de estrógeno estão muito aumentados e caem depois do parto, e a perimenopausa, quando o estado hormonal encontra-se caótico. Sendo a progesterona antagonista do estrógeno em alguns tecidos, é provável que ela neutralize os efeitos positivos deste hormônio sobre o humor das mulheres. Contudo, a deficiência estrogênica no humor não se encontra bem definida (Freitas \& Pimenta, 2006; Hanan, Magalhães \& Hanan, 2001; Stahl, 2002).

Miller, Conney, Rasgon, Fairbanks e Small (2002) desenvolveram um estudo para investigar diferenças sexuais no humor e na cognição e se os efeitos do estrógeno poderiam explicar parcialmente tais diferenças, conforme reportado em pesquisas prévias. A amostra foi composta por 96 adultos saudáveis com idades entre 57 e 75 anos (média=65,9), sendo, 31 mulheres usuárias de TRH (19 usavam estrógeno mais progesterona e as demais apenas estrógeno), 16 não usuárias e 49 homens. O nível educacional e o socioeconômico, bem como a idade, foram controlados. De acordo com os resultados, as mulheres do grupo TRH tiveram menos e mais leves sintomas de depressão que as não usuárias e os homens, conforme avaliados pela escala Profile of Mood States (POMS). Ainda, as mulheres usuárias de TRH demonstraram melhor fluência semântica (geração de palavras em uma categoria: frutas e vegetais) que os homens. O grupo TRH também se saiu melhor que o grupo não TRH em atenção e memória de trabalho (Dígitos WAIS-R), e neste caso, os homens se saíram melhor que as não usuárias. Os resultados não foram afetados pela duração do tratamento nem pelo tipo de medicação usada. Tais evidências apoiam a hipótese de que o estrógeno seja uma variável importante para explicar as diferenças de sexo no desempenho cognitivo e no humor na velhice.

\section{Considerações Finais}

Durante a meia-idade, a mulher passa por um processo longo e gradual de declínio na produção dos 
hormônios femininos conhecido como climatério, sendo a menopausa (última menstruação) um dos fatores que ocorrem dentro deste período. O climatério tem consequências sobre a saúde e a cognição que se tornaram mais evidentes com o aumento da expectativa de vida. Por isso, atualmente, muitas mulheres fazem administração exógena de hormônios para alívio dos sintomas típicos desta fase de suas vidas.

Para tratar os sintomas do climatério é indicado o uso da TRH. Mas, apesar de ser um tratamento antigo e eficaz no combate aos fogachos, ressecamento da mucosa urogenital e prevenção da osteoporose, ainda não desfruta de consenso em relação aos seus efeitos sobre a saúde da mulher.

Se a TRH ainda não dispõe de consenso entre os especialistas em relação aos seus efeitos sobre a saúde física, quando se trata dos seus benefícios sobre o Sistema Nervoso Central não é diferente. Do ponto de vista fisiológico, é perfeitamente compreensível que tal tratamento se relacione com a cognição devido aos receptores de estrógeno localizados nas mais diversas áreas cerebrais. No entanto, as pesquisas sobre o tema nem sempre encontram uma associação entre desempenho cognitivo e uso da TRH.

É importante destacar também que não há consenso sobre as habilidades cognitivas que são positivamente influenciadas pela TRH. Os estudos reportados neste artigo apontam uma relação entre a TRH e habilidades cognitivas bastante distintas, desde aquelas relacionadas à inteligência fluida (memória visual e habilidade espacial) até aquelas relacionadas à inteligência cristalizada (fluência semântica, memória e raciocínio verbal).

Além do mais, a dificuldade de consenso na área deve-se à heterogeneidade das pesquisas, devido às inúmeras variáveis relacionadas ao tema. Tanto a idade de início do tratamento quanto a idade dos participantes da amostra parecem influenciar os resultados. 0 tipo de medicação usada, bem como a dosagem e a via de administração, também são variáveis relevantes. A duração do tratamento e a distância entre a idade da menopausa e o início da TRH também podem influir nos resultados dos estudos. Portanto, são necessárias mais pesquisas com o intuito de elucidar os benefícios desta terapia, a influência dos hormônios no envelhecimento, a distinção das habilidades mais e menos suscetíveis às mudanças hormonais que acompanham a menopausa e outros fatores associados à menopausa e à TRH que possam também influenciar a cognição.

Internacionalmente, há um grande interesse pelo tema. No Brasil, não foram encontradas pesquisas sobre o assunto. Assim, o desenvolvimento de investigações que permitam aprofundar a compreensão da relação entre hormônios sexuais e cognição poderá contribuir para a melhoria da qualidade de vida das mulheres, particularmente na terceira idade.

\section{Referências}

Almeida, L. S. (2002). As aptidões na definição e avaliação da inteligência: o concurso da análise fatorial. Paidéia: Cadernos de Psicologia e Educação, 12 (23), 5-17.

Andrés-Pueyo, A. (2006). Modelos psicométricos da inteligência. In C. Flores-Mendoza \& R. Colom (Eds.), Introdução à psicologia das diferenças individuais (pp.73-100). Porto Alegre: Artmed.

Camarano, A. A. (2006). Envelhecimento da população brasileira: uma contribuição demográfica. In E. V. Freitas, L. Py, F. A. X. Cançado, J. Doll \& M. L. Gorzoni (Eds.), Tratado de geriatria e gerontologia (pp.88-105). Rio de Janeiro: Guanabara Koogan.

Chaimowicz, F. (2006). Epidemiologia e o envelhecimento no Brasil. In E. V. Freitas, L. Py, F. A. X. Cançado, J. Doll \& M. L. Gorzoni (Eds.), Tratado de geriatria e gerontologia, (pp.106-129). Rio de Janeiro: Guanabara Koogan.

Colom, R. (2006). O que é inteligência? In C. Flores-Mendoza \& R. Colom (Eds.), Introdução à psicologia das diferenças individuais (pp.59-72). Porto Alegre: Artmed.

Erkkola, R. (1996). Female menopause, hormone replacement therapy, and cognitive processes. Maturitas, 23 (Supl. 1), 27-30.

Flores-Mendoza, C., \& Nascimento, E. (2001). Inteligência: o constructo melhor investigado em psicologia. Boletim de Psicologia, 51 (114), 37-64.

Freitas, E. V., Pimenta, \& L. (2006). Climatério. In E. V. Freitas, L. Py, F. A. X. Cançado, J. Doll \& M. L. Gorzoni (Eds.), Tratado de geriatria e gerontologia (pp.766-775). Rio de Janeiro: Guanabara Koogan.

Hanan, M. Z., Magalhães, D. R. B., \& Hanan, B. Z. (2001). Climatério. In A. F. Camargos \& V. H. Melo (Eds.), Ginecologia ambulatorial (pp.233-254). Belo Horizonte: Coopmed.

Haskell, S. G., Richardson, E. D., \& Horwitz, R. I. (1997). The effect of estrogen replacement therapy on cognitive function in women: a critical review of the literature. Journal of Clinical Epidemiolog, 50 (11), 1249-1264.

Kimura, D. (2002). Sex hormones influence human cognitive pattern. Neuroendocrinology Letters, 23 (Suppl. 4), 67-77.

Liberman, S. (2006). Envelhecimento do sistema endócrino. In E. V. Freitas, L. Py, F. A. X. Cançado \& J. Doll, M. L. 
Gorzoni (Eds.), Tratado de geriatria e gerontologia (pp.758-765). Rio de Janeiro: Guanabara Koogan.

Magalhães, D. R. B., Hanan, M. Z., \& Magalhães, E. B. (2001). Geriatria em ginecologia. In A. F. Camargos \& V. H. Melo (Eds.), Ginecologia ambulatorial (pp.255-274). Belo Horizonte: Coopmed.

Maki, P. M. (2006). Hormone therapy and cognitive function: is there a critical period for benefit? Neuroscience, 138 (3), 1027-1030.

Maki, P., \& Hogersvorst, E. (2003). HRT and cognitive decline. Best Practice\&Research Clinical Endocrinology\&Metabolism, 17 (1), 105-122.

McEwen, B. S., Alves, S. E., Bulloch, K., \& Weiland, N. G. (1997). Ovarian steroids and the brain: Implications for cognition and aging. Neurology, 48 (Suppl. 7), 8-15.

Miller, K. J., Conney, J. C., Rasgon, N. L., Fairbanks, L. A., \& Small, G. W. (2002). Mood symptoms and cognitive performance in women estrogen users and nonusers and men. Journal of the American Geriatrics Society, 50 (11), 1826-1830.

Nascimento, E. (2000). Adaptação e validação do teste wais-iii para um contexto brasileiro. Tese de doutorado não-publicada, Universidade de Brasília.

Neisser, U., Boodoo, G., Bouchard, T. J., Boykin, A. W., Brody, N., Ceci, S. J., et al. (1996). Intelligence: knowns and unknowns. American Psychologist, 51 (2), 77-101.

Neri, A. L. (2006). Envelhecimento cognitivo. In E. V. Freitas, L. Py, F. A. X. Cançado, J. Doll \& M. L. Gorzoni (Eds.), Tratado de geriatria e gerontologia (pp.1236-1244). Rio de Janeiro: Guanabara Koogan.

Perls, T. \& Fretts, R. C. (2007). Por que as mulheres vivem mais que os homens. Scientific American: Ciência e Saúde, 1(Edição especial), 88-93.
Piato, S. (2005). Distúrbios da pós-menopausa. In S. Piato (Ed.), Terapêutica endócrina em ginecologia (pp.101-114). São Paulo: Artes Médicas.

Rodrigues, N. C. \& Rauth, J. (2006). Os desafios do envelhecimento no Brasil. In E. V. Freitas, L. Py, F. A. X. Cançado, J. Doll \& M. L. Gorzoni (Eds.), Tratado de geriatria e gerontologia (pp.186-192). Rio de Janeiro: Guanabara Koogan.

Rozestraten, R. J. A. (2002). Evolução inicial dos estudos da inteligência do idoso: mitos e fatos. Paidéia: Cadernos de Psicologia e Educação, 12 (23), 149-162.

Sherwin, B. B. (2005). Estrogen and memory in women: how can we reconcile the findings? Hormones and Behavior, 47 (3), 371-375.

Sherwin, B. B. (2006). Estrogen and cognitive aging in women. Neuroscience, 13 (3), 1021-1026.

Spirduso, W. W. (2005). Questões de quantidade e qualidade de vida. In W. W. Spirduso (Ed.), Dimensões fisicas do envelhecimento (pp.5-34). Barueri: Manole.

Stahl, S. M. (2002). Psicofarmacologia relacionada ao sexo e à função sexual. In S. M. Stahl (Ed.), Psicofarmacologia (pp.527-556). Rio de Janeiro: Medsi.

Verghese, J., Kuslansky, G., Katz, M. J., Sliwinski, M., Crystal, H. A., Buschke, $H_{\text {., }}$ et al. (2000). Cognitive performance in surgically menopausal women on estrogen. Neurology, 55 (6), 872-874.

Recebido em: 14/2/2008

Versão final reapresentada em: 24/4/2009

Aprovado em: 26/5/2009 